\title{
CDISC SDTM Fitzpatrick Skin Classification Terminology
}

National Cancer Institute

\section{Source}

National Cancer Institute. CDISC SDTM Fitzpatrick Skin Classification Terminology. NCI

Thesaurus. Code C74560.

Terminology codelist used for the Fitzpatrick Skin Classification Scheme within the Clinical Data Interchange Standards Consortium Study Data Tabulation Model. 